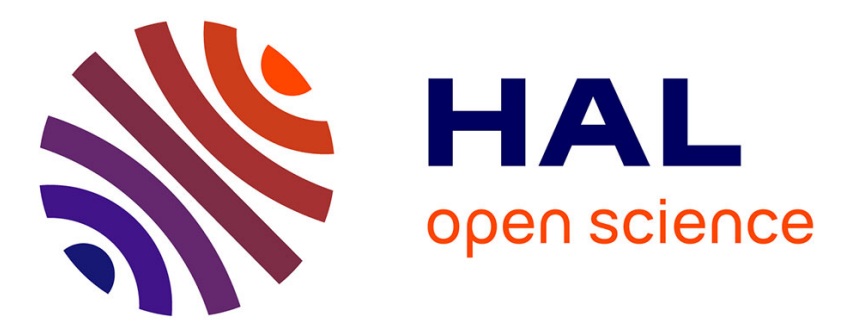

\title{
Dynamiques institutionnelles et conflit autour des droits d'eau dans un système d'irrigation traditionnel au Maroc
}

\author{
Paul Mathieu, Ahmed Benali, Olivia Aubriot
}

\section{To cite this version:}

Paul Mathieu, Ahmed Benali, Olivia Aubriot. Dynamiques institutionnelles et conflit autour des droits d'eau dans un système d'irrigation traditionnel au Maroc. Revue Tiers Monde, 2001, 42 (166), pp.353-374. 10.3406/tiers.2001.1509 . hal-00595983

\section{HAL Id: hal-00595983 \\ https://hal.science/hal-00595983}

Submitted on 13 Feb 2018

HAL is a multi-disciplinary open access archive for the deposit and dissemination of scientific research documents, whether they are published or not. The documents may come from teaching and research institutions in France or abroad, or from public or private research centers.
L'archive ouverte pluridisciplinaire HAL, est destinée au dépôt et à la diffusion de documents scientifiques de niveau recherche, publiés ou non, émanant des établissements d'enseignement et de recherche français ou étrangers, des laboratoires publics ou privés. 


\section{Dynamiques institutionnelles et conflit autour des droits d'eau dans} un système d'irrigation traditionnel au Maroc

Paul Mathieu, Ahmed Benali, Olivia Aubriot

\section{Citer ce document / Cite this document :}

Mathieu Paul, Benali Ahmed, Aubriot Olivia. Dynamiques institutionnelles et conflit autour des droits d'eau dans un système d'irrigation traditionnel au Maroc. In: Tiers-Monde, tome 42, $n^{\circ} 166,2001$. Les nouvelles politiques de l'eau. Enjeux urbains, ruraux, régionaux. pp. 353-374;

doi : 10.3406/tiers.2001.1509

http://www.persee.fr/doc/tiers_1293-8882_2001_num_42_166_1509

Document généré le 25/05/2016 


\title{
DYNAMIQUES INSTITUTIONNELLES ET CONFLIT AUTOUR DES DROITS D'EAU DANS UN SYSTÈME D'IRRIGATION TRADITIONNEL AU MAROC ${ }^{1}$
}

\author{
par Paul Mathieu* Ahmed Benali** et Olivia Aubriot***
}

L'article présente les effets d'une intervention externe à la fois technique (réhabilitation des réseaux) et institutionnelle (création d'associations d'usagers de l'eau) dans un système d'irrigation semitraditionnel du Nord du Maroc. Cette intervention a défini des enjeux nouveaux et induit des tensions, puis un processus conflictuel de négociation et de recherche d'un compromis acceptable pour toutes les parties, mais surtout pour les groupes dominants. A la lumière de cette analyse, le texte discute ensuite quelques questions de portée plus générale concernant les dynamiques institutionnelles des systèmes irrigués paysans : le lien entre investissement et droits d'appropriation, les fondements idéologiques des mécanismes de coordination, ainsi que les relations entre conflit et changement des institutions de gestion de l'eau.

Une intervention technique externe qui transforme un système d'irrigation traditionnel soulève de nombreuses questions qui se rap-

* Professeur, Université catholique de Louvain, Institut d'études du développement, et chercheur qualifié du FNRS. Il est aussi membre de l'uR "Régulations foncières » et membre associé de l'uR "Dynamiques sociales de l'irrigation » de l'IRD.

** Superviseur des Associations d'usagers de l'eau (AUEA), projet de mise en valeur du périmètre Ghiss-Nekkor (Maroc). Sa contribution à cet article est exprimée à titre personnel et n'engage pas son institution d'appartenance.

*** Agronome et anthropologue, UPR 299, CNRS. Sa contribution à cette recherche a bénéficié d'un appui de l'AUF (bourse d'excellence à l'UCL, en 1998-1999).

1. Le point de départ de ce texte est une communication présentée en juin 2000 à la conférence de l'Association internationale pour l'étude de systèmes de propriété communautaire (IASCP : International Association for the Study of Common Property) à Bloomington (États-Unis), dans le cadre d'une session consacrée à l'irrigation. La première partie de l'article reprend, sous une forme révisée, une partie du texte initial à paraître dans International Journal of Water (2001).

Revue Tiers Monde, t. XLII, n 166, avril-juin 2001 
portent aux changements matériels mais aussi sociaux qu'elle entraîne. Ainsi l'investissement exogène entraîne-t-il le plus souvent une modification physique de la distribution de l'eau qui elle-même provoque, de façon implicite ou explicite, une redéfinition du partage de la ressource et donc aussi des droits sous-jacents à ce partage. Or, cette modification du partage de l'eau suscite des enjeux et questions multiples: appropriation de l'eau, redéfinition des règles d'irrigation, répartition des revenus liés à l'opération d'aménagement, redéfinition des positions de pouvoir au sein du système d'irrigation et vis-à-vis des représentants de l'État, etc. Ces enjeux liés dépassent la seule question du partage de l'eau : ils sont enchâssés dans les rapports sociaux et les institutions sociales de gestion de l'eau. À partir d'une étude de cas, le présent texte discute diverses questions qui nous semblent centrales dans les processus de transformation volontariste des systèmes irrigués traditionnels.

Comment expliquer, par exemple, qu'une telle opération entraîne parfois de l'inertie ou des réactions conflictuelles, et dans d'autres cas des solutions de consensus favorisant l'action collective et entraînant l'adhésion de la majorité des usagers ? Quels sont les principes fondamentaux qui doivent être partagés et acceptés de tous pour qu'une action collective puisse se réaliser ? Comment la rareté de l'eau et même la compétition pour celle-ci peuvent-elles motiver des processus de négociation et d'innovation institutionnelle menant à des compromis et un certain consensus?

La question des conflits pour l'eau d'irrigation est particulièrement complexe et importante. Les situations de concurrence locale sont en effet nombreuses et sans doute de plus en plus en plus fréquentes. Elles entraînent, dans un certain nombre de cas, des confrontations plus ou moins violentes'. Ces dynamiques conflictuelles sont centrales dans les transformations des formes d'organisation sociale pour la gestion des ressources naturelles ${ }^{2}$. Mais la concurrence n'entraîne pas toujours le conflit, et le conflit ne devient pas toujours violent : la négociation est une autre modalité particulière de conflit, lorsqu'il y a " communication dans l'affrontement $"^{3}$ et recherche d'un compromis. À la suite de divers travaux récents qui ont étudié les processus de négociation et de compétition autour des droits d'eau ${ }^{4}$, nous essaierons

1. Pour quelques exemples, voir Bedoucha (1987), Kilani (1986), Hunt et Hunt (1974), Bolin (1990), Ouhajou (1996).

2. Chauveau et Mathieu, 1998

3. Suivant l'expression de Bailey, 1971 . (2000)

4. Pradhan et Pradhan (1996), Bruns et Meinzen-Dick (2000), von Benda-Beckmann et Pradhan 
de voir dans un cas particulier comment la rareté et la compétition ont pu aller de pair avec une négociation qui a permis la recherche de nouveaux arrangements institutionnels.

Une autre question se rapporte au lien existant entre investissement et droits d'eau dans les pratiques de partage de l'eau entre agents du système d'irrigation'. Depuis près de vingt ans, en effet, avec les travaux de Coward ${ }^{2}$, diverses études ont mis en lumière la relation essentielle qui unit la définition des droits d'eau et l'investissement pour la création du réseau d'irrigation, dans les systèmes irrigués traditionnels. En résumé : ceux qui ont investi pour la construction du réseau - puis leurs descendants - sont reconnus comme "propriétaires de l'eau ", ayant le droit de prendre certaines parts d'eau, et considérés comme détenteurs légitimes de ces droits d'eau. Dans ce contexte, comment une intervention extérieure - qui représente un nouvel investissement et complique donc la "donne » initiale - est-elle comprise et intégrée par les utilisateurs du réseau d'irrigation?

Dans la première partie de ce texte, nous présentons l'histoire de la seguia Boulamaiz dans la plaine de Ghiss, au nord du Maroc. Après une intervention de modernisation du réseau et de création d'associations d'usagers de l'eau, ce système d'irrigation a été, entre 1993 et 1995 , le théâtre d'un double conflit. Le premier opposait les propriétaires des terres et eaux de l'amont, traditionnellement considérés comme seuls propriétaires légitimes des "droits d'eau ", et ceux de l'aval, arrivés tardivement en bordure d'un aménagement déjà créé par les premiers. Un second désaccord a opposé les mêmes paysans de l'amont à l'encadrement technique de l'État, à propos des institutions de gestion du réseau. La deuxième partie de l'article offre une brève analyse des fondements des droits d'eau traditionnels à la lumière de l'étude de cas qui précède. Enfin, la dernière partie propose des éléments de réponses aux questions posées ci-dessus, en formulant quelques hypothèses et pistes de recherche plus générales pour l'analyse des relations entre droits d'eau, conflit et changement institutionnel dans les systèmes irrigués.

1. Ambler, 1990.

2. Voir, notamment, Coward, 1986 et 1990. 
I. UNE ÉTUDE DE CAS AU NORD DU MAROC: LE PERIMĖTRE DE GHISS ${ }^{1}$

\section{Décalages dans les perceptions d'un espace hydraulique}

Dans le nord du Maroc, A. Benali a étudié les aménagements traditionnels d'une surface irriguée totale de 920 ha, qui capturent et utilisent les eaux de l'oued Ghiss dans le nord-est de la région du Rif, à proximité de la ville d'Al Hoceima. Ces réseaux traditionnels, déjà en partie modernisés par des canaux primaires et secondaires bétonnés sous la colonisation espagnole vers 1930, étaient fortement dégradés dans les années 1980. Dans le cadre d'un projet financé par la FAO, ils ont bénéficié d'une réhabilitation technique (réfection des réseaux et des prises d'eau en rivière) entre 1989 et 1991, suivie en 1992 de la mise en place de trois associations d'usagers de l'eau agricole (AUEA), destinées à gérer les trois réseaux correspondant à chacun des trois canaux principaux.

Pour la mise en place de ces associations, la cellule technique responsable de la mise en valeur des périmètres, qui relevait des services régionaux de l'agriculture, a procédé en 1991-1992 à un recensement des irrigants ou usagers de l'eau, conformément à la nouvelle loi sur les associations d'usagers de l'eau ${ }^{2}$. Ce recensement identifiait un nombre total de 479 usagers pour la seguia (canal traditionnel principal) de Boulamaiz, 410 et 449 usagers pour les deux autres seguias. À partir de ce moment, une situation ambiguë s'est progressivement créée dans la seguia la plus grande (Boulamaiz) pour aboutir à un conflit ouvert fin 1994 et en 1995.

Au point de départ de ce conflit, il y a eu notamment un décalage entre les perceptions des intervenants extérieurs et celles des acteurs locaux. L'entité spatiale et agraire irrigable par le réseau hydraulique dépendant de cette seguia renvoyait en fait à des significations territoriales et des réalités sociales très différentes dans les perceptions des techniciens et des paysans.

a) Pour les premiers, responsables des études de réhabilitation, puis cadres agricoles de l'État, le réseau Boulamaiz du périmètre de

1. Le récit et une partie de l'analyse présentée dans cette partie reprennent des informations présentées de façon plus extensive dans le travail de Benali, 2000.

2. La loi $n^{\circ} 02.84$ relative à la création des associations d'usagers des eaux agricoles (AUEA) a été adoptée par la Chambre des représentants (Parlement) en février 1984. Le Dahir (décret d'application) portant promulgation de cette loi est daté du 21 décembre 1990, et il a été effectivement diffusé début 1991 par le Bulletin officiel $(B O) n^{\circ} 4081$ du 16 janvier 1991, entraînant à ce moment la relance de la mise en place d'associations qui n'existaient jusque-là que sur papier. 
Ghiss représentait, après réhabilitation, une seule entité hydraulique caractérisée par une interdépendance amont-aval et une source principale, unique, d'approvisionnement en eau. Comme entité hydroagricole, la zone irrigable du réseau Boulamaiz était simplement définie comme la mise en relation d'un espace cultivable et d'une disponibilité en eau : une surface totale de 480 ha cultivés pour 479 usagers de l'eau, vus comme égaux sinon identiques.

b) Ces mêmes usagers, quant à eux, distinguaient clairement deux catégories sociales d'exploitants et deux parties bien distinctes du «territoire sociohydraulique ", par rapport à l'appropriation de l'eau distribuée par la seguia Boulamaiz.

Après être resté un long moment latent, un double conflit autour des droits d'eau a éclaté lorsque ce décalage entre perceptions est apparu au grand jour. Celui-ci opposait d'une part le groupe des détenteurs des droits d'eau coutumiers, les ayants droit, aux nondétenteurs de ces droits, et d'autre part les premiers (les ayants droit) à l'administration. Du point de vue historique et social de l'appropriation de l'eau, une distinction claire existait en effet entre la zone amont ( $250 \mathrm{ha})$ aménagée par les lignages fondateurs du réseau, et la zone aval "résiduelle" pour ce qui concerne le contrôle de l'eau. La zone amont était appropriée et contrôlée par 319 détenteurs de droits d'eau, descendants des lignages fondateurs et dont les droits ont été transmis par héritage ; la zone aval était cultivée par 160 exploitants propriétaires de ces terres aval, mais ne détenant pas de droits coutumiers de propriété de l'eau de la seguia Boulamaiz. Considérés par la coutume comme ne possédant pas de «droits de propriété de l'eau ", c'est-à-dire des parts d'eau précisément définies, identifiées au sein d'un tour d'eau nommé, attaché au nom d'un lignage, les paysans de l'aval ont cependant un droit d'usage socialement admis sur les quantités résiduelles parvenant en aval. Précisons ici qu'au Maroc, l'expression "droits d'eau " n'est utilisé qu'en référence aux droits séculaires de propriété, acquis antérieurement à la loi de 1914'. Les droits d'usage des paysans de l'aval ne sont donc pas des droits d'eau, au sens strict de l'usage de l'expression au Maroc.

Alors que ce clivage fondamental des droits d'appropriation de l'eau était évident et fondamental pour les paysans, les concepteurs du projet de réhabilitation et les techniciens agricoles ne l'ont pas intégré ni reproduit dans la réhabilitation technique et le schéma d'organisation des usagers, puisque le nouveau réseau allait en principe four-

1. Voir Bouderbala (1992), El Alaoui (1979). 
nir de l'eau en suffisance pour tous ${ }^{1}$. Cette question est donc longtemps restée de l'ordre du non-dit, sans être prise en compte de façon explicite dans la démarche d'aménagement et d'organisation.

\section{Droits d'eau et gestion sociale de la rareté}

Dans le système traditionnel de gestion et d'appropriation de l'eau tel qu'il fonctionne dans cette région, les ayants droit se définissent et sont reconnus comme propriétaires des droits d'eau. Ces droits correspondent à des durées d'irrigation de fractions de débit de canal. La quantité d'eau qu'ils permettent de délivrer est donc variable, en fonction de l'abondance de l'eau dans le réseau à chaque moment. Il s'agit d'une "eau célibataire " ${ }^{2}$, car les droits d'eau ne sont pas attachés au foncier, ils ne sont pas liés à des parcelles spécifiques ou proportionnels à leur surface. Ils peuvent alors être hérités indépendamment des terres, de même qu'échangés ou cédés temporairement pour aller arroser une autre parcelle. Cette mobilité du droit d'eau dans l'espace reste cependant limitée et n'a aucun caractère marchand: elle est d'ordre social et communautaire (réciprocité, entraide, héritage) et ne peut pas être objet d'un échange monétaire. "L'eau du ciel (véhiculée par la seguia) ne peut pas être vendue", disent les paysans. Une telle transaction ferait l'objet d'une réprobation sociale unanime : elle est donc très rare, et lorsqu'elle se produit, elle est soigneusement dissimulée.

Au contraire, l'eau provenant des pompages individuels dans la nappe, grâce à de petites motopompes achetées individuellement par certains paysans, surtout dans la zone aval, se vend ouvertement pour l'irrigation, à des prix clairement signalés, et cela sans aucune contrainte ni réprobation sociale. En dehors de l'eau provenant de la seguia et contrôlée par les détenteurs des droits d'eau, les cultivateurs de l'aval ont en effet accès à une autre ressource hydrique complémentaire : le forage dans la nappe phréatique, depuis 1985 surtout. Les quantités d'eau résiduelles pour la zone aval étant trop incertaines et insuffisantes durant les périodes les plus sèches, ces exploi-

1. D'autre part, dans les réseaux d'irrigation implantés par l'État, l'eau est sous le régime de la domanialité et ne peut être objet d'appropriation privative individuelle. Les droits d'eau en tant que propriété n'y existent pas ou ne sont pas reconnus. Voir, à ce sujet, Zirari, 1995, p. 99-100. La loi de 1995 apporte aussi des restrictions aux droits d'eau coutumiers qui ne sont pas «mariés" (liés) à la terre. Ces droits ne peuvent être cédés qu'avec la propriété du fonds, et les détenteurs de droits d'eau isolés ne peuvent les céder qu'à des propriétaires fonciers (Zirari, op. cit., p. 101).

2. Zirari, op. cit. 
tants sont contraints de trouver des apports complémentaires ou de se limiter aux cultures les moins exigeantes en eau.

L'irrigation des semis de céréales, principale culture de la majorité des exploitants, est généralement assurée par deux arrosages d'eaux de crue en septembre ou octobre. En année climatique "normale", ces crues sont assurées et les eaux suffisantes pour inonder à la fois amont et aval, zone des ayants droit et zone des non-détenteurs de droits d'eau. Par la suite, les eaux de pluie prennent le relais pour la croissance des céréales, et c'est surtout au printemps et en été, pour la fin des céréales, les cultures fourragères (luzerne, bersim) et le maraîchage, qu'une certaine rareté de l'eau se manifeste. À ce moment, les quantités d'eau disponibles, parfois très limitées, sont distribuées suivant les tours d'eau coutumiers, avec une mesure très rigoureuse des droits d'eau. La zone amont utilise donc à ce moment la plus grande part de l'eau disponible, seuls les débits résiduels étant laissés à la zone aval.

Concrètement, la gestion traditionnelle de l'eau est donc fondée sur un partage de l'eau clairement défini et accepté entre les propriétaires et non-propriétaires de droits d'eau. La mise en cuvre effective des droits d'eau reste cependant flexible et à "géométrie variable ", avec une répartition des droits au jour le jour qui se traduit par un partage plus ou moins strict ou flexible, suivant les saisons et les circonstances. Le caractère fluctuant et variable du rapport entre disponibilité de la ressource en eau et besoins d'irrigation ( $"$ offre " et demandes en eau) est une caractéristique centrale de ce système d'irrigation, et cette variabilité est fonction des moments de l'année, des années plus ou moins sèches, et des producteurs (choix culturaux à l'intérieur du système de production). Pour répondre aux besoins variables des uns et des autres, il arrive fréquemment que des quantités d'eau soient cédées en cadeau ou en échange (eau contre travail) entre divers irrigants proches, surtout lorsque ceux-ci sont parents ou alliés. Cette circulation plus souple de l'usage de l'eau est totalement imbriquée dans les relations sociales traditionnelles : les exploitants définissent eux-mêmes ces échanges comme l'effet d'une relation de "bonne entente ". La gestion sociale du réseau traditionnel (surveillance des infractions, organisation des travaux d'entretien annuels) est supervisée par la Jmaa, le conseil traditionnel des notables. Les différends sont portés devant le juge coutumier (Kadi abouri), qui recherche d'abord une solution socialement acceptable aux litiges entre irrigants. C'est seulement en cas d'échec de cette première démarche que les désaccords sont portés devant les tribunaux.

Dans ce système, les droits d'eau inégaux reflètent la structure sociale et sont en principe reconnus par tous, y compris par ceux n'en 
possédant pas. Moyennant la reconnaissance et l'acceptation de l'inégalité, les droits d'eau sont concrètement mis en œuvre de façon souple et négociable, au cas par cas, en fonction des besoins. Le principe structurant et stable des droits d'eau peut être appliqué de façon variable, mais il reste toujours subordonné à une conditionnalité sociale : l'existence de "bonnes relations " entre les personnes concernées, avec tout le caractère asymétrique de cette relation; les exploitants en amont et en tête de réseau ont la possibilité matérielle de prélever un maximum d'eau en ne laissant que des quantités résiduelles à ceux qui sont en aval. L'un a besoin de l'eau appartenant à l'autre, et doit donc assumer les conditions de la dite bonne entente. L'échange hydraulique reproduit ainsi la structure des rapports de dépendance sociale et économique : les exploitants de la zone aval dépendent des ayants droit qui contrôlent l'eau, à la fois socialement et physiquement, en amont. La flexibilité de la mise en œuvre concrète des droits d'eau, tout comme l'apparente " générosité » des détenteurs de ceux-ci, sont possibles parce que le principe hiérarchique de ces droits est reconnu par tous comme stable, légué par l'histoire, idéologiquement intégré et constitutif des positions sociales.

En période de rareté et de pénurie d'eau, et toujours moyennant cette même conditionnalité des rapports sociaux, les règles des tours d'eau sont parfois "adaptées ", comme si elles étaient d'abord des points de repères indicatifs autour desquels les usagers proches ou interdépendants se débrouillent comme ils l'entendent. En période d'extrême sécheresse, les tours d'eau peuvent même être modifiés pour sauver une partie des cultures d'une majorité d'exploitants, à l'intérieur de la zone amont et du groupe des ayants droit. Dans ce système, les droits d'eau codifiés par la tradition ont donc clairement vocation à ne pas être appliqués de façon rigide et inconditionnelle : la règle coutumière appelle une adaptation, de commun accord entre les irrigants proches, en fonction des besoins des cultures et de la situation hydrologique, fort variable d'une année à l'autre. En temps normal, les règles coutumières de distribution sont même souvent ouvertement contournées, et ces infractions acceptées, à condition de rester dans certaines limites et dans le cadre du consensus communautaire sur les "droits d'eau » des uns et l'absence de "droits d'eau » des autres. Cette relation dialectique entre stabilité des principes et souplesse des transactions effectives apparaît centrale dans la gestion de l'eau.

Ce système a fonctionné de façon relativement stable et harmonieuse jusqu'en 1993 : la différence des statuts sociaux d'appropriation de l'eau étant clairement établie et acceptée, les droits d'eau inégaux 
reflétaient une réalité sociale admise comme évidente. Le consensus social de l'ensemble de la communauté autour du système d'irrigation ne signifiait cependant pas que le partage inégal pour l'appropriation de l'eau était une donnée entièrement acceptée et figée une fois pour toutes. En 1995, quatre ans après la fin de la réhabilitation technique et deux ans après la mise en place formelle des associations, un épisode de sécheresse a entraîné la remise en cause du consensus de gestion de l'eau entre amont et aval, entre détenteurs coutumiers et non détenteurs des droits d'eau.

\section{Affrontement et changement institutionnel négocié autour des droits d'eau: conflit, compromis, innovation}

Fin 1992, l'association du réseau Boulamaiz est officiellement constituée, après une période relativement brève d'information par les techniciens de l'agriculture, qui s'adressent surtout à des paysans jugés représentatifs de l'ensemble des usagers. Vu l'impossibilité d'obtenir une participation de plus de $20 \%$ des usagers aux assemblées générales', l'administration de l'agriculture choisit de faire élire des représentants des usagers au niveau des canaux secondaires et tertiaires, de façon sommairement proportionnelle : une personne élue par les usagers d'une maille hydraulique représente alors un groupe d'une douzaine d'usagers à l'assemblée générale. Cette assemblée désigne, fin 1992, un conseil d'administration composé de sept membres. D'après la loi sur les AUEA et leur règlement intérieur type, les dirigeants de l'association ont la responsabilité d'assurer l'entretien des réseaux et la gestion de l'eau, notamment par la définition des tours d'eaux en période de pénurie. L'association est reconnue officiellement comme l'interlocuteur institutionnel qui représente les usagers, et son conseil d'administration compte un membre de l'administration locale de l'agriculture. Celle-ci continue d'ailleurs, durant la phase de démarrage de l'AUEA, à lui prodiguer un encadrement rapproché, des conseils et divers appuis techniques. Comme les ayants droit, dans l'ensemble plus aisés, sont aussi plus absentéistes ${ }^{2}$ que les nondétenteurs de droits d'eau coutumiers, le premier conseil d'administration de l'AUEA, élu en 1992, compte une majorité d'usagers de l'aval, non détenteurs de droits d'eau coutumiers.

1. Alors que le quorum officiellement requis est de $2 / 3$ des membres au minimum, lors de la première convocation d'une assemblée générale.

2. Ils migrent, ou ont une activité principale autre que l'agriculture. 
Entre mai 1992, date de création, et décembre 1994, troisième assemblée générale, l'association de Boulamaiz existe formellement, sans problèmes ni tensions apparents, mais un peu comme une coquille vide, sans guère de réalisations concrètes. Considérant que la réhabilitation doit accroître les débits disponibles et ainsi permettre l'irrigation de toute la zone alimentée par le réseau, soit les 480 ha, les membres du Conseil d'administration, appuyés par les services d'encadrement officiels, gèrent l'eau pendant ces années-là suivant une logique égalitaire. Les tours d'eau décidés assurent les quantités nécessaires à la majorité des usagers, y compris ceux de l'aval, sans plus se référer de façon explicite aux droits d'eau traditionnels. Cela ne pose pas de problème en 1992 et 1993, années de pluies abondantes, où l'administration reste relativement proche de la gestion directe du réseau, notamment par le travail de deux aigadiers rémunérés par elle pour assurer la fermeture et l'ouverture des vannes des canaux primaires et pour organiser les tours d'eaux. On peut supposer que la majorité des propriétaires de droits d'eau sont restés ignorants du changement officiel dans les règles d'appropriation et de partage de l'eau, car peu intéressés par l'AUEA et moins présents sur le périmètre : un grand nombre d'entre eux font en effet exploiter leur terres par de la maind'œuvre salariée, par des métayers ou des locataires.

À la troisième assemblée générale, en octobre 1994, et à un moment qui coïncide avec le début d'une année très sèche dans tout le Maroc, les propriétaires de droits d'eau sont pour la première fois présents en nombre et découvrent, ou font mine de découvrir, que le fonctionnement effectif des tours d'eau, depuis deux ans, ne tient plus compte des droits d'eau traditionnels. Lors de cette assemblée générale, trois positions s'expriment :

a) Les représentants des non-détenteurs de droits d'eau coutumiers (un usager aval, membre du Conseil d'administration de l'AUEA) : « La colonisation est finie, nos agronomes ont tranché l'affaire, l'extension du périmètre a été faite jusqu'à nos portes, et le temps de deux poids, deux mesures : c'est terminé. La répartition de l'eau depuis la création de l'association a été très équitable et elle devra le rester. "

b) Les ayants droit (un vieil homme, en réponse à l'orateur cidessus) : "Cela est le mauvais résultat de cette association. Jamais et depuis mon enfance, personne n'a eu l'arrogance de dire ce qui vient d'être dit. " Et un autre : «Ce que nous voulons, c'est simplement la reconnaissance de nos droits d'eau comme il en était avant l'association, et nous ne comprenons pas "prioritaires", nous sommes propriétaires. " Cette dernière réplique venait en réponse à la proposition de conciliation de l'administration (ci-dessous). 
c) L'administration : elle tente vainement, durant la réunion, de concilier les points de vue en distinguant droit d'eau (traditionnel) et droit d'usage (acquis par les usagers aval à la suite de l'extension du périmètre), en précisant que les ayants droit traditionnels, en amont, restaient prioritaires.

Cette position de compromis ne satisfait visiblement pas les ayants droit et, entre octobre 1994 et mars 1995, ceux-ci - qui représentent, rappelons-le, près de deux tiers des usagers du périmètre et de la surface irriguée -, boycottent l'association et refusent de participer à ses activités. En même temps, ils s'organisent en vue de faire pression directement auprès de l'administration pour la dissolution d'une association regardée par eux comme illégitime et inacceptable, puisqu'elle considère comme usagers de l'eau, au même titre qu'eux-mêmes, " des gens qui n'ont jamais été propriétaires de l'eau ".

Entre octobre 1994 et mars 1995, les requêtes et plaintes des ayants droits n'aboutissent à aucun résultat, l'administration insistant pour que la solution soit trouvée dans le cadre de l'association d'usagers définie par la loi comme le lieu de décision pour les différends sur la gestion de l'eau. En mars 1995, excédés par ce qu'ils vivent comme une "expropriation » encore plus insupportable durant une année de sécheresse aiguë, un groupe d'une trentaine d'ayants droits se rend à la prise d'eau sur l'oued Ghiss. À l'aide d'outils manuels et d'un tracteur, ils dévient l'eau dans l'ancien chenal d'adduction qui se déversait directement dans l'ancien réseau, toujours existant; l'eau peut alors être distribuée à partir des anciens répartiteurs et des ouvrages traditionnels de la partie amont, donc dans le réseau correspondant aux droits d'eau et aux tours d'eau anciens.

Après cet affrontement violent, les dégâts et la déviation sont réparés par les services techniques agricoles, mais ceux-ci prennent aussi l'initiative d'une négociation élargie entre les deux groupes rivaux sous l'égide d'autorités locales en partie externes au débat : l'administration locale et communale et certains notables traditionnels. Dans ce cadre, une solution de compromis d'urgence est négociée, dont les principaux éléments sont les suivants ${ }^{1}$.

- Reconnaissance des droits d'eau traditionnels.

- Limitation de ces droits à un volume (débit) correspondant aux débits avant réhabilitation. De cette façon, le surplus d'eau résultant directement de l'investissement de l'État pourrait être affecté à la zone aval du périmètre au bénéfice des non-détenteurs de droits d'eau. 
- Obligation aux ayants droits d'utiliser eux-mêmes leurs droits d'eau. Autrement dit, l'attributaire absent ou qui n'utilise pas effectivement sa part d'eau au moment voulu ne peut pas décider lui-même de la céder à quelqu'un d'autre. Ces droits d'eau non utilisés viennent donc en principe accroître le surplus qui peut être affecté à l'irrigation de la partie aval.

- Les questions de la représentation des usagers et de la désignation des dirigeants de l'association doivent être tranchées dans le cadre institutionnel défini par la loi, c'est-à-dire par une assemblée générale de l'AUEA en vue d'élire de nouveaux représentants et un nouveau conseil d'administration.

- L'administration de l'agriculture, pour sa part, s'engage à rechercher d'autres sources d'alimentation en eau pour la partie aval, afin de compenser le déficit qui ne manquerait pas de réapparaître pour cette zone si on reprenait les tours d'eau tels que définis par les droits d'eau traditionnels.

Ce compromis est négocié entre les dirigeants des deux groupes et en présence de diverses autorités locales et régionales durant la seconde moitié de l'année 1995. Début 1996, l'assemblée générale de l'AUEA, où les représentants des ayants droit sont cette fois majoritaires, désigne un nouveau conseil d'administration contrôlé par eux. Cette même assemblée approuve le compromis réglant le problème des droits d'eau entre ayants droit et non-détenteurs de droit séculaire. Concrètement, ces décisions ont pour conséquence un " retour au système traditionnel de distribution de l'eau sur la base des droits d'eau [traditionnels] reconnus par la communauté ${ }^{\prime}$. On peut essayer de détailler cette appréciation en distinguant les principaux changements suivants au terme de ces diverses péripéties conflictuelles, entre 1992 et 1996 :

- le volume d'eau total disponible a augmenté, et le surplus doit en principe bénéficier à l'aval, qui reste cependant globalement déficitaire ;

- la forme institutionnelle de la gestion de l'eau a changé, ce qui entraînera peut-être dans l'avenir une transformation notable des modes de gestion de l'eau;

- les rapports de pouvoir réels qui organisent l'appropriation effective de l'eau ne sont guère modifiés par rapport à la situation avant réhabilitation. Les groupes dominants gardent le pouvoir de définition et de mise en œuvre des droits d'eau qu'ils détenaient auparavant, mais ce pouvoir est maintenant « encadré » et transposé dans 
le cadre d'une institution formelle. Celle-ci (l'AUEA) est imposée par l'État, et elle obéit à des règles définies par la loi. Le cadre institutionnel formel des pratiques a changé, mais le contenu du cadre - le tissu même des relations et des pratiques sociales de gestion de l'eau - semble relativement inchangé jusqu'ici.

\section{LES FONDEMENTS DE LA GESTION TRADITIONNELLE DE L'EAU}

\section{Investissement et appropriation :}

une relation idéologique structurante

Le principe du lien direct et essentiel entre investissement dans la création du réseau et propriété de l'eau semble bien central dans cette structuration stable, tant qu'elle est socialement légitime et acceptée, des rapports sociaux et hydrauliques. On le retrouve dans la plupart sinon dans toutes les sociétés rurales où l'irrigation joue un rôle important : l'investissement initial fonde les droits d'eau, et les relations de propriété ainsi définies entre les acteurs constituent la base sociale de l'action collective de gestion du système irriguél. Cette liaison idéologique est forte dans la plupart des systèmes traditionnels d'irrigation ; elle est donc « évidente » et elle établit un lien à première vue irrécusable entre l'investissement initial, ou historique, réalisé par un groupe et les droits de propriété sur l'eau que s'est approprié, par ce geste, le groupe en question. Cette liaison fonde un partage (appropriation inégale) de la ressource qui est bien établi et socialement stabilisé, c'est-à-dire légitime et prévisible, très difficile à remettre en cause, même si le partage est inégal. La distribution effective de l'eau, qui actualise ce partage, fonctionne à travers des institutions et des règles qui se présentent comme l'expression de ce principe fondant les droits d'eau. Le rapport de propriété par rapport à l'eau se traduit par un mécanisme d'inclusion/exclusion : les uns sont membres du groupe des ayants droit et doivent être reconnus comme tels par l'ensemble de la communauté ; les autres sont exclus, ne sont pas membres de ce groupe et doivent s'accepter comme tels. À cette condition, la bonne entente sociale leur permettra d'accéder aux volumes d'eau excédentaires, non utilisés par les ayants droit. 


\section{Coordination, stabilité et flexibilité}

Le système social de coordination de la distribution de l'eau repose sur une dialectique qui associe stabilité et flexibilité. La stabilité des principes concernant les droits permet la souplesse de la transaction sociale au jour le jour pour l'usage et la distribution de l'eau. Cette relation structure tout le système de gestion de la ressource. Le cadre de référence stable de l'interaction est défini par les rapports politiques d'autorité et par les rapports idéologiques d'appropriation de la ressource eau. À l'intérieur de ce cadre - qui définit des balises fixes de l'appropriation -, le pilotage effectif des transactions interindividuelles dispose d'une réelle marge de manœuvre et se caractérise par la souplesse des pratiques concrètes de répartition de l'eau. C'est par rapport au cadre de référence des droits d'eau que les acteurs du système irrigué gèrent la rareté de l'eau, cette gestion consistant à décider au jour le jour comment distribuer et répartir celle-ci, en fonction des besoins de chacun et des relations entre acteurs, dans le contexte ressources/besoins contingent de chaque moment.

La coordination et les transactions quotidiennes de répartition de l'usage de l'eau se gèrent ici de façon relativement ouverte et flexible. Cette dynamique contractuelle est négociée de façon bilatérale entre acteurs. Elle fonctionne cependant nécessairement, et uniquement, de façon conditionnelle, subordonnée à la dimension relationnelle (la " bonne entente ») et dans le cadre stable des droits d'appropriation des eaux. La convention sociale fondamentale est stable et elle porte sur l'appropriation, ainsi que sur la hiérarchie des statuts et des positions de décisions pour la gestion de cette ressource. Les transactions et les ajustements de type contractuel sont flexibles et temporaires, et ils portent sur l'usage de la ressource, non sur son appropriation durable'.

1. La distinction entre dimension conventionnelle et dimension contractuelle d'un arrangement institutionnel est suggérée par Colin, 1998. 
III. QUELQUES HYPOTHĖSES ET PISTES DE RÉFLEXION :

INVESTISSEMENT, DROITS D'EAU, INSTITUTIONS

ET DYNAMIQUES SOCIALES

\section{Stabilité et élasticité des rapports d'appropriation et des mécanismes de coordination de la gestion de l'eau}

Les droits d'eau, perçus comme principe de base des rapports sociaux du système d'irrigation, permettent ainsi à la fois d'organiser le partage des droits d'appropriation de la ressource et de fonder les relations entre acteurs ayant un accès différentiel à celle-ci. Une coordination des opérations de répartition de l'eau est indispensable dans tout système d'irrigation, dès lors que cette eau n'est pas assez abondante pour que tous les usagers puissent l'utiliser à leur guise à tout moment. Les coûts sociaux de transaction jouent aussi un rôle important: ils peuvent vite devenir insupportables dans un système d'irrigation où les comportements opportunistes sont très répandus. Une telle situation entraîne en effet des dépenses considérables de temps, d'énergie et/ou de disputes pour la surveillance et pour la formalisation des accords entre irrigants.

Il est important que la base idéologique de ces mécanismes de coordination soit stable, car sa remise en cause incessante rendrait très difficiles et socialement coûteuses les opérations de gestion de l'eau. Un principe structurant clair et légitime peut ainsi être à la base d'une coordination efficace, les relations de propriété définies par ce principe constituant la base sociale de l'action collective de gestion du système irrigué. Un principe social légitime qui définit les bases des relations et des mécanismes de coordination remplit diverses fonctions essentielles, même s'il est socialement vécu comme relevant des valeurs et des normes, et non de l'utilité fonctionnelle. Il permet en effet de stabiliser le cadre de référence des interactions concrètes (pour le partage et la distribution de l'eau), d'atténuer les tensions entre irrigants, et enfin de modérer, voire pacifier, les modalités et rythmes de transformation du système d'irrigation. Toutes ces fonctions permettent de minimiser les coûts de transaction. Cette stabilité des principes fondant les règles et normes coutumières du partage de l'eau doit cependant laisser une certaine souplesse de mise en application des normes, dans la pratique. Cette flexibilité est nécessaire pour un fonctionnement collectif efficace, qui puisse intégrer la variabilité des besoins et des situations.

En outre, les règles de gestion de l'eau découlant du principe fondamental peuvent être sujettes à négociations et ajustements. La rela- 
tion sociale et idéologique (investissement $\rightarrow$ droits sur la ressource) n'est effectivement pas donnée une fois pour toutes, de façon intemporelle et incontestable après l'événement initial : elle peut évoluer et intégrer de nouveaux rapports sociaux, comme cela a été le cas pour la seguia Boulamaiz. On peut penser que cette transformation s'est réalisée de façon relativement efficace pour deux raisons : d'une part, la clarté du principe fondateur des droits d'eau, avec les intérêts, droits et obligations en résultant pour chaque partie prenante, et d'autre part, la stabilité des relations sociales rendant acceptables et légitimes ces obligations et droits respectifs de chaque groupe. Le processus de production d'un compromis par les différents groupes impliqués dans la gestion de la seguia Boulamaiz apparaît donc lié à la très forte imprégnation idéologique de ce principe structurant (investissement / droits d'eau) et de ses conséquences sociales.

\section{Projet et interventions exogènes : interférences, enjeux emboîtés, risques et opportunités}

La relation idéologique fondatrice (investissement/rapports de propriété) de la ressource fait que « l'investissement d'un agent externe dans l'infrastructure d'irrigation est un acte créateur de propriété qui interfère avec les relations de propriété [de l'eau] antérieures ${ }^{1}$. Dès lors que l'un des éléments constitutifs de l'aménagement traditionnel a été modifié par une intervention externe (État, projet, réhabilitation, définition de nouvelles institutions de gestion), l'appropriation inégale de la ressource peut être remise en cause par les membres du groupe défavorisé.

Dans le cas de la seguia de Boulamaiz, tant que les relations sociales locales restaient globalement stables et aussi longtemps que la base à la fois technique et idéologique du réseau d'irrigation restait inchangée, avant réhabilitation et constitution d'une AUEA, les relations sociales par rapport à l'eau (rapports sociohydrauliques) et les institutions de gestion de l'eau sont restées quasi immuables. Pour les non-détenteurs de droits d'eau coutumiers, l'intervention extérieure représentait l'occasion de remettre en cause ces droits d'eau et de profiter d'un partage plus égalitaire de la ressource, sur la base d'une autre légitimité imposée de l'extérieur, si l'administration avait appuyé jusqu'au bout leurs revendications. Un tel changement aurait bien sûr amélioré radicalement leur situation sociale, dans cette société où

1. G. Gerbrandy et P. Hoogendam, 1996, p. 55. 
«l'eau est l'amie du puissant "1, en leur assurant un certain «droit" ou contrôle sur l'eau d'irrigation et en leur permettant d'occuper une place dans les nouvelles institutions de gestion de l'eau.

En tant qu'investissement et modification exogène de l'infrastructure d'irrigation, l'intervention d'aménagement crée un moment d'incertitude : comment vont être redéfinies les règles du jeu d'appropriation et de gestion de la ressource, puisqu'un des éléments reconnu comme structurant du jeu est soudainement modifié, sans un accord préalable explicite sur les conséquences distributives de cette modification ? Ce moment est donc aussi une occasion et une " ouverture " soudainement accrue de potentialités de changement social : un moment favorable, ou même obligé, pour orienter la redéfinition des règles, des statuts, des rapports de pouvoir, bref des diverses interactions entre les acteurs du système d'irrigation.

L'intervention externe définit de nouveaux enjeux au sein des communautés locales, c'est-à-dire des " occasions de gagner ou de perdre quelque chose ". Des options habituellement fermées apparaissent soudain ouvertes, rendant possibles des changements éventuels dans les équilibres et les rapports de pouvoir. L'intervention sur l'infrastructure entraîne des conséquences sur la structure sociale : en modifiant un élément, à première vue simplement technique, du système matériel qui organise le contrôle et la distribution de l'eau, l'intervention oblige à redéfinir un aspect des règles du jeu social par rapport à la gestion et l'appropriation de cette ressource. Pour les acteurs qui ont intérêt à certains changements, c'est le moment de tenter de modifier les règles du jeu, afin d'imposer ou de stabiliser de nouvelles règles qui améliorent leur situation.

Entre les paysans de statuts sociaux différents et/ou situés à divers endroits du réseau, l'enjeu au premier degré et le plus visible, c'est de savoir qui pourra avoir les quantités d'eau souhaitées au moment voulu. Cet enjeu renvoie aussitôt à d'autres enjeux de pouvoir au second degré.

Il y a donc deux principaux types d'enjeux créés par l'opération d'aménagement $: a$ ) la répartition de l'eau par la définition des droits d'eau ; b la légitimité, la stabilité et/ou la recomposition des équilibres de pouvoir dans les institutions de gestion de l'eau. Ces deux groupes d'enjeux sont en fait liés et interdépendants.

Les enjeux de pouvoir se situent à leur tour à deux niveaux, l'un interne à la communauté ou à l'espace local, le second engageant l'articulation entre le local et l'extérieur: l'État, les institutions 
d'aide, les espaces de pouvoir plus larges dans lesquels sont définis les projets.

a) Entre différents groupes ruraux cohabitant dans le même espace social, l'enjeu est «la manière dont un groupe social définit son identité (...), les processus par lesquels il établit ses relations avec d'autres groupes à l'intérieur d'un espace d'interactions ${ }^{\prime}{ }^{1}$.

b) Entre l'État, représenté par ses techniciens du génie rural et ses administrateurs locaux, et les paysans, " des deux côtés, le risque est gros, car il s'agit de savoir qui, en définitive, sera maître chez lui $\aleph^{2}$. L'enjeu est ici la répartition des pouvoirs respectifs de l'État et des acteurs paysans dans la définition des règles et dans la mise en ouvre effective des rapports de contrôle des ressources : eau, taxes et redevances, ressources financières diverses circulant dans les institutions de gestion de l'irrigation, prises de décision.

\section{Le conflit comme communication et innovation institutionnelle}

Il serait sans doute réducteur de voir le conflit comme un événement seulement négatif et perturbateur, provoqué par des méthodes inadaptées ou par la seule méconnaissance de la complexité du social par les intervenants extérieurs. Sous un autre angle, le conflit peut aussi être vu comme un processus de communication plutôt que simplement comme un désaccord reflétant une divergence d'intérêts et entraînant une suite d'événements malheureux. Les diverses étapes du conflit ont été un processus de confrontation, mais aussi une négociation des équilibres de pouvoirs entre divers groupes. Cette confrontation a permis de produire finalement une innovation institutionnelle acceptée par tous. On pourrait suggérer qu'une bonne solution du conflit - une innovation institutionnelle efficiente et durable - n'a été possible qu'après cette «bonne communication conflictuelle». À l'occasion de cette "communication dans l'affrontement", chaque acteur a pu affirmer ses objectifs et ses capacités et apprécier ceux des autres, optimiser sa position dans les rapports de force nouveaux, mesurer et affirmer aussi les limites de l'acceptable et du non négociable, bref "mettre sur la table " les divers paramètres à partir desquels on pourra éventuellement aboutir à un compromis acceptable par toutes les parties prenantes. 
L'innovation institutionnelle qui résout le conflit de la seguia Boulamaiz repose finalement sur une nouvelle définition formalisée des droits d'eau, qui ne remet cependant pas en cause les droits d'eau séculaires. Cette redéfinition des droits et, implicitement, des relations est considérée comme acceptable par toutes les parties prenantes lorsqu'elle apparaît en phase avec l'équilibre des pouvoirs en présence et cohérente avec l'image mentale (idéelle, idéologique) de l'objet social "système d'irrigation ». Le nouveau cadre institutionnel apparaît opérationnel aux usagers et à l'État, il peut donc constituer une nouvelle base de coordination acceptable. La stabilité de ce cadre n'a pu être obtenue que lorsque le contenu effectif de la nouvelle structure institutionnelle exogène a été adapté pour être mis en harmonie avec les relations sociales et les rapports de pouvoir locaux. C'est à ce moment, et à ce moment seulement, que le cadre institutionnel de l'AUEA a été accepté, de façon plus ou moins volontaire, par tous les acteurs du système d'irrigation.

L'innovation institutionnelle négociée a consisté en fait à produire un nouveau cadre institutionnel formel permettant de satisfaire les principales exigences des deux groupes d'acteurs dominants : d'une part, les représentants de l'État qui veulent que "force reste à la loi », au moins dans les formes et les procédures, et d'autre part le groupe des paysans de l'amont, ayants droit traditionnels qui veulent dominer dans les prises de décision concernant le système d'irrigation'. Ces deux groupes ont enfin trouvé une base de compromis permettant à chacun de préserver des avantages importants tout en cédant sur une partie de ce qui était essentiel pour l'autre ${ }^{2}$. La trajectoire du conflit montre l'évolution possible d'un système d'irrigation à travers diverses étapes : inertie, conflit d'abord verbal, confrontation, violence, institutionnalisation d'un dialogue, négociation et compromis.

\section{CONCLUSION}

L'histoire de la seguia Boulamaiz nous montre aussi que l'aménagement irrigué est un objet sociotechnique complexe : à la fois espace physique et territoire social, support de la production agricole

1. On observe ici, comme dans de nombreux autres cas (cf. Aubriot et Jolly, 2001), que l'apparition d'une nouvelle règle ne remet pas fondamentalement en cause les précédentes.

2. Cette production d'un "compromis pratique " illustre un processus fondamental dans de très nombreuses interactions humaines (Goffman, 1988, p. 102). 
et lieu de reproduction des rapports sociaux ${ }^{1}$. Dans cette reproduction, les rapports d'appropriation de l'eau jouent un rôle déterminant. Le conflit a montré que l'espace hydraulique était un territoire sociopolitique signifiant et pas seulement une étendue physique à mettre en valeur. L'aménagement hydro-agricole se révèle ici comme étant à la fois la structure matérielle qui opère la circulation et la distribution matérielle de l'eau, et un objet technique socialement construit, structuré par les rapports d'appropriation de l'eau. Le système irrigué était et reste un lieu, ou un lien, social, fonctionnant selon une logique de territoire, c'est-à-dire « la relation entre un espace, une autorité locale et un groupe localisé ${ }^{2}$. Ce territoire apparaît organisé et contrôlé de façon asymétrique et complexe, loin de l'homogénéité et de l'égalité que voulaient instaurer l'Etat et les aménageurs. L'intervention étatique n'a pas pu modifier l'asymétrie d'appropriation de la ressource qui reflète ${ }^{3}$ elle-même des rapports sociaux inégalitaires, car le principe structurant des rapports d'appropriation de l'eau a été maintenu de façon vigilante par le groupe dominant et est resté intégré, voire accepté par l'ensemble des irrigants.

BIBLIOGRAPHIE

Ambler J. S. (1990), The influence of farmer water rights on the design of water-proportioning devices, in Design Issues in Farmer-Managed Irrigation Systems : Proceedings of an International Workshop held at Chiang Mai, Thaïlande, 12-15 décembre 1989, Colombo, Sri Lanka, IIMI, p. 3752.

Aubriot O. (1997), Eau : miroir des tensions. Ethno-histoire d'un système d'irrigation dans les moyennes montagnes du Népal central, Université de Provence (Aix-Marseille I), thèse de doctorat en anthropologie, $602 \mathrm{p}$., multig. (version révisée à paraître, 2002).

Aubriot O. (2001), Société et concept de droits d'eau en irrigation : appropriation ou partage de l'eau ?, in $\mathrm{O}$. Aubriot et $\mathrm{G}$. Jolly (éd.), Histoires d'une eau partagée. Irrigation et droits d'eau du Moyen Áge à nos jours, Pyrénées, Alpes, Provence, Aix-en-Provence, Publication de l'Université de Provence.

Bailey F.-G. (1971), Les règles du jeu politique, Paris, PUF.

Bedoucha G. (1987), L'eau, l'amie du puissant : une communauté oasienne du Sud-tunisien, Paris, Éd. des Archives contemporaines.

1. Mathieu, 1995.

2. Gruenais, 1986, p. 291.

3. Sur l'eau comme reflet des rapports sociaux, voir Aubriot, 1997. 
Benali Ahmed (2000), Aménagement hydro-agricole et gestion participative. Le cas du périmètre de Ghiss au nord du Maroc, Louvain-la-Neuve (Belgique) : mémoire de DEA en développement, population et environnement, Louvainla-Neuve, UCL, Dept of Population and Development Studies, 94 p. + ann.

Bolin I. (1990), Upsetting the power balance : cooperation, competition and conflict along an Andean irrigation systems, Human Organization, 49 (2), 140148.

Bouderbala N. (1992), Maroc, in Les périmètres irrigués en droit comparé africain (Madagascar, Maroc, Niger, Sénégal, Tunisie), FAO (éd.), Étude législative $\mathrm{n}^{\circ} 48$, Rome, FAO, p. 58-59.

Bruns B. R. et R. Meinzen-Dick (Eds) (2000), Negotiating Water Rights, New Delhi, Sage.

Chauveau J.-P. et P. Mathieu (1998), Dynamiques et enjeux des conflits fonciers, in $\mathrm{Ph}$. Lavigne-Delville (éd.), Quelles politiques foncières pour l'Afrique noire rurale?, Paris, Karthala, p. 243-258.

Colin J.-P. (1998), Institutions agraires et pratiques foncières. Méthodologie d'une recherche sur les modes de faire-valoir au Mexique, à paraître dans Institutions agraires et pratiques foncières. Une étude comparée des contrats agraires au Mexique.

Coward E. Walter Jr. (1986), Direct or indirect alternatives for irrigation investment and the creation of property, in $\mathrm{K}$. William Easter (ed.), Irrigation Investment, Technology and Management Strategies for Development. Boulder, CO, Westview Press.

Coward E. Walter Jr. (1990), Property Rights and Network Order: The Case of Irrigation Works in the Western Himalayas, Human Organization, 49 (1), p. 78-88.

El Alaoui M. (1979), Aspects du régime juridique de la propriété, de l'exploitation et de la gestion des eaux et des réseaux d'irrigation au Maroc, Hommes, terre et eaux, $\mathrm{n}^{\circ} 33$, p. 39.

Funnell D. C. (1994), Intervention and indigenous management. The geography of small-scale irrigation development in Morocco and Swaziland, Land Use Policy, 11 (1), p. 45-54.

Gerbrandy G. et P. Hoogendam (1996), The materialization of water rights. Hydraulic property in the extension and rehabilitation of two irrigation systems in Bolivia, in G. Diemer, F. P. Huibers (Eds), Crops, People and Irrigation. Water allocation practices of farmers and engineers, Londres, Intermediate Technology Publications, p. 53-72.

Goffman E. (1988), L'ordre social et l'interaction, in Les moments et leurs hommes (textes réunis et présentés par Y. Winkin), Paris, Éd. du Seuil / Minuit.

Gruenais M. E. (1986), Territoires autochtones et mise en valeur des terres, in B. Crousse, E. Le Bris, E. Le Roy (éd.), Espaces disputés en Afrique noire. Pratiques foncières locales, Paris, Karthala.

Hunt E. et R. C. Hunt (1974), Irrigation, conflict and politics. A Mexican case, in T. E. Downing and McGuire Gibson (éd.), Irrigation's impact on 
society. Anthropological papers of the University of Arizona, $\mathrm{n}^{\circ} 25$, Tucson, Arizona, États-Unis, The University of Arizona Press, p. 129-157.

Kilani Mondher (1986), L'influence de l'État dans la transformation du système hydraulique du groupe d'oasis de Gafsa (Tunisie), Genève-Afrique, 24 (2), p. 7-46.

Kilani Mondher (1994), L'eau et le lignage dans l'oasis d'El Ksar. Sur la notion d'ethnicité, in L'invention de l'autre. Essais sur le discours anthropologique, Genève, Payot, p. 206-235.

Laurent P. J. et P. Mathieu (1995), Compétition foncière et invention sociale locale. Un exemple au Burkina Faso, in A. Bertrand, E. Le Roy et A. Karsenty (éd.), La sécurisation de la terre, Paris, Karthala, p. 286-303.

Mathieu P. (1995), Social relationships and effective irrigation projects. Two sahelian experiences, Irrigation and Drainage Systems, $\mathbf{n}^{\circ}$ 9, p. 39-58.

Ouhajou L. (1996), Espace hydraulique et société au Maroc. Cas des systèmes d'irrigation de la vallée du Dra Agadir, Université Ibn Zohr, Publ. de la Faculté des lettres et sciences humaines, série Thèses et Mémoires, $344 \mathrm{p}$.

Pradhan R. et U. Pradhan (1996), Staking a claim : law, politics and water rights in farmer managed irrigation systems in Nepal, in J. Spiertz et M. Wibes (Eds), 1996, The role of law in natural resource management, The Hague, VUGA, p. 61-76.

Sautter G. (1987), Libres réflexions sur les aménagements ayant pour objet la maîtrise de l'eau par ou pour les agriculteurs, in Aménagements hydroagricoles et systèmes de production, Montpellier, CIRAD, Documents systèmes agraires $n^{\circ} 6, t$. I, p. 13-23.

Trottier J. (1999), Hydropolitics in the West Bank and Gaza strip, Jérusalem, Passia Publications, $246 \mathrm{p}$.

Von Benda-Beckmann F. et K. et Rajendra Pradhan (2000), Introduction, in $\mathrm{R}$. Pradhan, F. et $\mathrm{K}$. von Benda-Beckmann (Eds), 2000, Water, land and law : changing rights to land and water in Nepal, Kathmandu, FreedealWAU-EUR, $278 \mathrm{p}$.

Zirari M. (1995), Maroc. La loi sur l'eau du 16 août 1995, in Yearbook of Islamic and Middle Eastern Law (vol. 2), E. Cotran et C. Mallat (Eds), Londres, Kluwer Law International. 